\title{
AN OPTICAL LINEWIDTH STUDY OF A CHROMOPROTEIN: C-PHYCOCYANIN IN A LOW TEMPERATURE GLASS
}

\author{
W. KÖHLER, J. FRIEDRICH \\ Physikalisches Institut und Bayreuther Institut für Makromolekülforschung (BIMF), Universität Bayreuth, \\ D-8580 Bayreuth, Federal Republic of Germany
}

\author{
R. FISCHER and H. SCHEER \\ Botanisches Institut, Universität München, D-8000 Munich 19, Federal Republic of Germany
}

Received 23 December 1987; in final form 22 February 1988

\begin{abstract}
The temperature dependence of spectral holes burnt into a phycocyanin-doped ethylene glycol/water glass is investigated in the temperature range between 1.5 and $15 \mathrm{~K}$. The data are well described by a power law with an exponent of $1.16 \pm 0.1$. Chromoproteins thus behave very much the same as glasses doped with small impurity molecules.
\end{abstract}

The temperature dependence of the linewidth of optical holes is very sensitive to the structure and order of the lattice to which a photoreactive probe molecule is coupled. Whereas in crystalline materials this temperature dependence involves a high power of $T$ or even an exponential, a rather low power law is characteristic for amorphous materials [1]. For many systems a power law with an exponent of 1.3 has been observed [2]. It should be stressed that the width of an optical hole may originate from several different processes such as direct electron-phonon scattering or phonon modulation of the electronTLS interaction. TLS stands for a special kind of twolevel system which is considered as the basic disorder mode of an amorphous glass. Its nature is not yet very clear, but it is assumed that some atoms or groups of atoms can occupy two different sites. The characteristic feature of glasses is that the eigenvalue splittings of the TLS are distributed flatly in a broad range extending close to zero [3]. Transitions between TLS are mediated by phonon-assisted tunneling. Concomitant with a distribution of the energy splitting is a distribution of tunneling relaxation rates. This dispersion of relaxation rates may, in turn, result in spectral diffusion processes which broaden the holes on time scales longer than the dephasing time. For several organic glasses we have shown that spec- tral diffusion may occur on time scales as long as days [4]. The question whether the width of an optical hole is determined by the dephasing time or by spectral diffusion processes is currently a subject of great interest. In any case the low power law originates from the presence of the TLS disorder modes and, hence, can be considered as a characteristic feature of glass-like disorder.

In this paper we present measurements of the spectral width of an optical hole burnt into a chromoprotein as a function of temperature in the range from 1.5 to $15 \mathrm{~K}$. A similar study was performed by Boxer et al. [5] for chlorophyllide in apomyoglobin. In these experiments, however, the temperature range investigated covered only $1.15 \mathrm{~K}$. In the following we will classify the spectral width as "quasi-homogeneous" in order to stress the fact that we do not know whether it is determined by spectral diffusion or not. The protein investigated is C-phycocyanin, a photosynthetic antenna pigment of the cyanobacterium, Mastigocladus laminosus. The monomer has a molecular weight of $37.4 \mathrm{kD}$. It contains three spectrally distinct chromophores whose component spectra in the visible band are known [6]. It can be safely assumed that the low energy chromophores, the socalled $\alpha-84$ and $\beta-84$ are degenerate within their inhomogeneous bands [6] but uncorrelated [7]. These 
chromophores are considered to be the fluorescing (f) ones and also those most active in hole burning. The higher energy chromphore, the so-called $\beta-155$ is blue-shifted by some $600 \mathrm{~cm}^{-1}$ and is considered to he the sensitizing one (s). The structure of the chromoprotein from two different species has been analyzed by $\mathrm{X}$-ray crystallography with a resolution limit of $2.1 \AA$. Hence not only the spectral but also the spatial location of the various chromophores is well known [8]. Usually, it is also assumed that the structure of the pigments in frozen solution is basically the same as in a crystal. It is the goal of this paper to determine whether the quasi-homogeneous width of an optical hole burnt into chromoproteins shows the same temperature dependence as holes burnt into doped glasses. In the sample investigated, C-phycocyanin is present as a trimer; hence the photoactive chromophores are partly shielded from the solvent molecules by the protein, and experience pronounced interactions with the protein [6]. Therefore an experiment of this kind may give information about the disorder modes in a protein. Glass-like behaviour has been shown for a few proteins at low temperatures, i.e. they roughly show a specific heat linear in temperature $[9,10]$ and show the same response to ultrasound and dielectric waves [10] as glasses.

Trimeric C-phycocyanin was isolated from Mastigocladus laminosus according to the method given in ref. [11]. It was dissolved in ethylene glycol/ water $(3: 1, v / v)$. Hole burning was achieved at $\lambda=6386 \AA$ with an $\mathrm{Ar}^{+}$pumped dye laser (Coherent $599)$ with a resolution of $10 \mathrm{MHz}$. The power level used was of the order of $10 \mu \mathrm{W} / \mathrm{cm}^{2}$. Burning times were in the range of seconds to minutes. All holes were detected in fluorescence [12]. Fig. 1 shows a typical series of holes as a function of the burning fluence. The quasi-homogeneous width was determined from extrapolating the linewidth data as a function of fluence to zero (fig. 1, inset). Usually the extrapolation was made from six to eight data points. The total dose was kept sufficiently low that the increase of the hole width with irradiation was linear. Fig. 2 shows the quasi-homogeneous width roughly measured over $10 \mathrm{~K}$ on a $\log -\log$ plot. The data points lie on a straight line with a slope of $1.16 \pm 0.1$. For the lowest temperature of $1.5 \mathrm{~K}$, we measured a width of $860 \mathrm{MHz}$. This value is by a factor of 5 larger

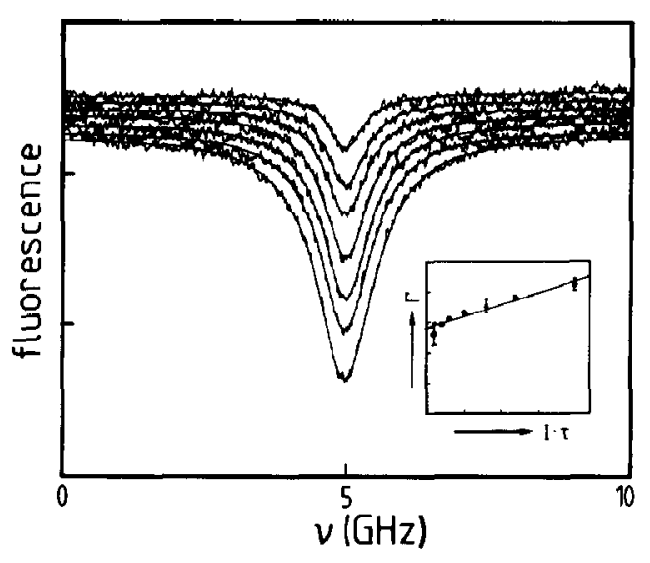

Fig. 1. A series of holes burnt at $1.6 \mathrm{~K}$ as a function of burning fluence. The laser intensity was $1.2 \mu \mathrm{W} / \mathrm{cm}^{2}$, burning times were between 80 and $1600 \mathrm{~s}$. The inset shows the corresponding linewidth data $(\Gamma)$ from which the quasihomogeneous width $\gamma(0.028$ $\mathrm{cm}^{-1}$ ) was obtained by a linear extrapolation to zero fluence. The $\Gamma$ scale is in units of $0.01 \mathrm{~cm}^{-1}$, the $I \tau$ scale in units of $\mathrm{mJ} / \mathrm{cm}^{2}$.

than the lifetime limit which is of the order of 100 $\mathrm{MHz}$ (1.6 ns).

This result shows that, as far as the optical linewidth is concerned, chromophores embedded in proteins behave at sufficiently low temperatures like dye molecules in amorphous polymers and glasses. A similar result was obtained from specific heat experiments on water-free samples of melanin and metmyoglobin $[9,10]$. The conclusion is that at least to some extent a protein resembles a glass. Its dynamics at low temperatures seems to be governed by

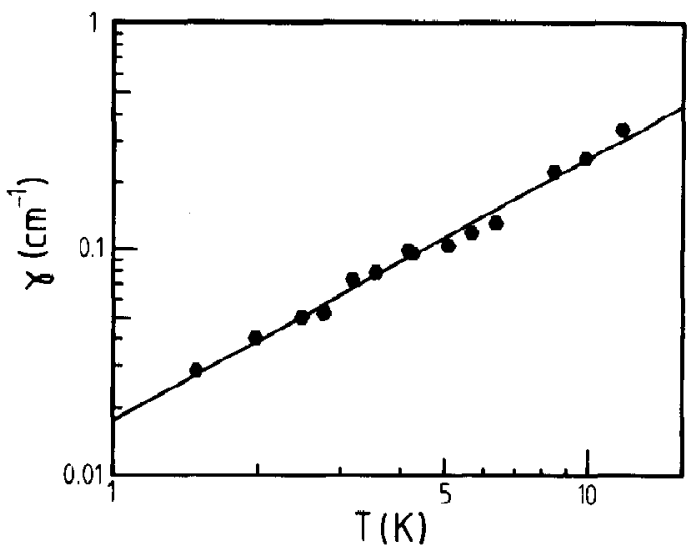

Fig. 2. Holewidth as a function of temperature. The straight line has a slope of $1.16 \pm 0.1$. For the fit procedure the residual width has been neglected. 
TLS-like excitations. Hence, on a microscopic level its structure is not well defined. In agreement with this conclusion is the fact that the optical lines are inhomogeneously broadened to a large extent and the various chromophore states are energetically decorrelated $[7,13]$. This latter phenomenon is very characteristic of glasses. For the chromoproteins investigated here it means that a frequency selective transition in a higher energy chromophore e.g. the $\beta$ 155 , is not frequency selective in the lower energy chromophores. Due to microscopic disorder the energy correlation is lost and the energy transfer processes populate most of the inhomogeneous band of the lower-lying states. This fact, together with the special level structure in C-phycocyanin, is responsible for the absence of any narrow lines in fluorescence despite sharp holes in absorption.

In summary we have shown that at low temperatures solid solutions of chromoproteins have much in common with impurity-doped glasses, though the chromophores are shielded from the glassy solvent to a high degree.

\section{Acknowledgement}

The authors acknowledge financial support from the Deutsche Forschungsgemeinschaft (SFB 213, Bayreuth; SFB 143 and Forschergruppe "Pflanzliche Tetrapyrrole", Munich).

\section{References}

[1] J. Friedrich and D. Haarer, Angew. Chem. 96 (1984) 96; Angew. Chem. Intern. Ed. Engl. 23 (1984) 113.

[2] H.P.H. Thijssen, R.E. van den Berg and S. Völker, Chem. Phys. Letters 97 (1983) 295.

[3] S. Hunklinger and A.K. Raychauduri, in: Progress in low temperature physics, Vol. 9, ed. D.F. Brewer (Elsevier, Amsterdam, 1986) p. 265.

[4] J. Fricdrich and D. Haarer, in: Optical spectroscopy of glasses, ed. I. Zschokke (Reidel, Dordrecht, 1986) p. 149.

[5] S.G. Boxer, D.S. Gottfried, D.J. Lockhart and T.R. Middendorf, J. Chem. Phys. 86 (1987) 2439.

[6] H. Scheer, in: Light rcaction path of photosynthesis, cd. F.K. Fong (Springer, Berlin, 1982) p. 7;

M. Mimuro, P. Füglistaller, R. Rümbeli and H. Zuber, Biochim. Biophys. Acta 848 (1986) 155;

K. Sauer, H. Scheer and P. Sauer, Photochem. Photobiol. 46 (1987) 427.

[7] W. Köhler, J. Friedrich, R. Fischer and H. Scheer, Chem. Phys. Letters 143 (1988) 169.

[8] T. Schirmer, W. Bode, R. Huber, W. Sidler and H. Zuber, Mol. Biol. 184 (1985) 257.

[9] I.-S. Yang and A.C. Anderson, Phys. Rev. B 34 (1986) 2942.

[10] G.P. Singh, H.J. Schink, H.V. Löhneysen, F. Parak and S. Hunklinger, Z. Physik B 55 (1984) 23.

[11] P. Füglistaller, H. Widmer, G. Frank and H. Zuber, Arch. Microbiol. 129 (1981) 268.

[12] H.P.H. Thijssen and S. Völker, Chem. Phys. Letters 120 (1985) 496.

[13] W. Köhler, J. Friedrich, R. Fischer and H. Scheer, in: Proceedings of Organization and Functioning of Photosynthetic Antennas, Freising 1987 (De Gruyter, Berlin, 1987). 